UDC 54.057;54.061;615.074;615.076.7

\title{
SYNTHESIS AND BIOLOGICAL ACTIVITY OF NEW DITHIOCARBAMATE DERIVATIVES
}

\author{
C.Y. Shukurov \\ Azerbaijan Medical University, Department of Pharmaceutical Chemistry \\ 167, S.Vurgun str., Baku, AZ 1022, Azerbaijan; e-mail: china.86@mail.ru
}

Received 15.01.2020

Accepted 13.05.2020

\begin{abstract}
Nowadays there are various antifungal agents. These included many substances, including derivatives of dithiocarbamates. The article aims to synthesize new derivatives of dithiocarbamate and study their antifungal activity. To analyze and identify synthesized substances and determine their structural formulas, the following spectral methods were used: IR (Shimadzu 8400 FTIR), IH NMR (Bruker $500 \mathrm{MHz}$ UltraShield) in DMSO-d6 (internal standard - tetramethylsilane), Mass spectroscopy (Agilent 1100 Series LC / MSD Trap VL \& $S L)$. Elemental analysis ( $C, H$ vo $N)$ was performed using a Perkin Elmer analyzer. The biological activity of synthesized derivatives of dithiocarbamates was explored and $100 \mu \mathrm{g} / \mathrm{ml}$ dilution of compound J2 (2-((4morpholinophenyl)amino)-2-oxoethyl 4-benzylpiperazine-1-carbodithioate) showed identical effect against Candida albicans, Candida crusei, Candida parapsilosis as fluconazole. Compound A4 (2-((4-(4methylpiperazin-1-yl)phenyl)amino)-2-oxoethyl-4-methylpiperazine-1-carbodithioate) showed the same activity against Candida parapsilosis and Candida glabrata as a standard drug.
\end{abstract}

Keywords: dithiocarbamate derivatives, antifungal activity, antibacterial activity

DOI: $10.32737 / 2221-8688-2020-2-174-180$

\section{Introduction}

Today there are many diseases caused by microorganisms. Some of these diseases are caused by the spread of pathogenic fungal infections. There are approximately 1.5 million different species of fungi on Earth, but just 300 of them are known to make people sick. Fungal diseases are often caused by fungi that are common in the environment [1].

A series of N-mono- and N, Ndisubstituted dithiocarbamates were investigated as inhibitors of three b-carbonic anhydrases (CAs, EC 4.2.1.1) from fungal pathogens Cryptococcus neoformans, Candida albicans and Candida glabrata, that is, Can2, CaNce103 and CgNce103, respectively. The new class of b-CA inhibitors may have potential for developing antifungal agents with a diverse mechanism of effect as compared to clinically used drugs for which drug resistance was reported [2].

Brug resistance for establishing antifungals, such as azole derivatives (fluconazole and ketoconazole), is a driving force in global mortality due to fungal infection. Identification of new molecular entities structurally unrelated to the above may represent a valid strategy to overcome resistance to currently available drugs. In an effort to develop highly potent antifungal agents, we report herein a series of 27 compounds of dithiocarbamate and rhodanine molecules containing pyridine moiety and their antifungal activity. Among synthesized compounds, several analogues showed potent antifungal activity [3].

A new series of Schiff bases of 4-(4aminophenyl)-morpholine were synthesized and described by IR, 1H-NMR, 13C-NMR, mass spectral and elemental analyses. The compounds were screened for antibacterial (Staphylococcus aureus (ATCC 9144), Staphylococcus epidermidis (ATCC 155), Bacillus cereus (ATCC 11778), Micrococcus luteus (ATCC 4678), 
Escherichia coli (ATCC 25922)), antifungal (Candida albicans (ATCC 2091) and Aspergillus niger (ATCC 9029)) activities [4-7].

Antibacterial activity was tested against Staphylococcus aureus (MTCCB 737), Pseudomonas aeruginosa (MTCCB 741), Streptomyces epidermidis (MTCCB1824) and Escherichia coli (MTCCB1652), as well as antifungal activity against Aspergillus fumigatus nigra Aspergillus.

A series of substituted piperazine derivatives were synthesized and tested for antimicrobial activity. Also, the antibacterial activity was tested against Staphylococcus aureus (MTCCB 737), Pseudomonas aeruginosa
(MTCCB 741), Streptomyces epidermidis (MTCCB1824) and Escherichia coli (MTCCB1652), and antifungal activity against Aspergillus fumigatus, Aspergillus flavus and Aspergillus niger. All synthesized compounds showed significant activity against bacterial strains but were found to be less active against tested fungi. In vitro toxicity tests revealed that some compounds showed lesser toxicity against human erythrocytes [5].

The purpose of our work is to take into the consideration of the main directions of research in the creation of new active, antifungal substances drugs of the future - including derivatives of dithiocarbamates.

\section{Materials and methods}

The substances cited were used as starting materials for synthesis of A4, A5, J1 and J2 compounds: N-methyl piperazine aniline (Great Britain Maybridge), 4-methyl piperazine, 4-ethyl piperazine, carbon disulfide, chloroacetyl chloride (Sigma Aldrich), sodium hydroxide, ethanol, 4morpholinoaniline (Great Britain Acros organics), 4-methyl 4-benzyl piperazine and 4benzyl piperazine, tetrahydrofuran (THF) as solvent and triethylamine (TEA) as catalyst.
To identify and analyze synthesized compounds and their structural formulas, spectral research methods were used: IR (Shimadzu 8400 FTIR), 1H NMR (Bruker 500MHz UltraShield) in DMSO-d6 (internal standart - tetramethylsilane) Mass Spectroscopy (Agilent 1100 Series LC / MSD Trap VL \& SL). Elemental analysis (C, H va N) was performed using Perkin Elmer analyzer.

\section{Results and discussion}

Synthesis of new derivatives of synthesis is reaction of acetylated 4dithiocarbamates with morpholine moiety was performed in three steps: acetylation of 4morpholineaniline with chloroacetyl chloride, synthesys of 4-methyl 4-piperazine-1dithiocarbamate and 4-benzylpiperazine-1dithiocarbamate sodium, final morpholineaniline with the synthesized 4-methyl 4-piperazine-1-dithiocarbamate and 4benzylpiperazine-1-dithiocarbamate sodium salts through obtaining basic substances (Scheme 1).

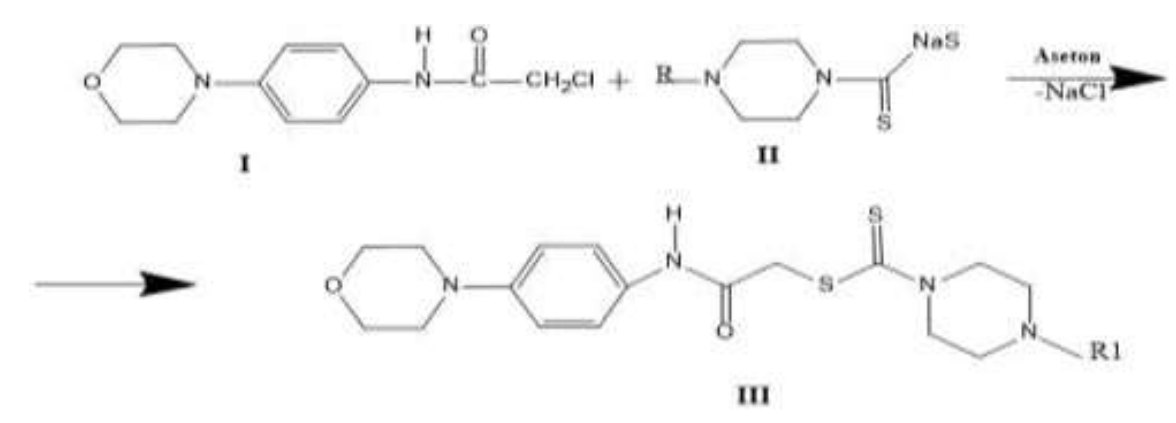




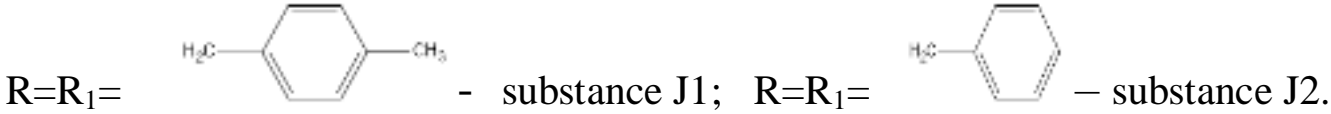

Scheme 1. Final step of the synthesis of dithiocarbamate dervatives (J1 and J2 compounds).

Synthesis of new derivatives of dithiocarbamate salts. Final synthesis was the dithiocarbamates with piperazine moiety was reaction of acetylated 4-piperazineaniline with the performed in three steps: acetylation of $\mathrm{N}$ - synthesized 4-methylpiperazine-1methylpiperazineaniline with chloroacetyl dithiocarbamate and 4 ethylpiperazine-1chloride, synthesys of 4-methylpiperazine-1- dithiocarbamate salts by the obtaining of basic dithiocarbamate and 4 ethylpiperazine-1- substances (Scheme 2).

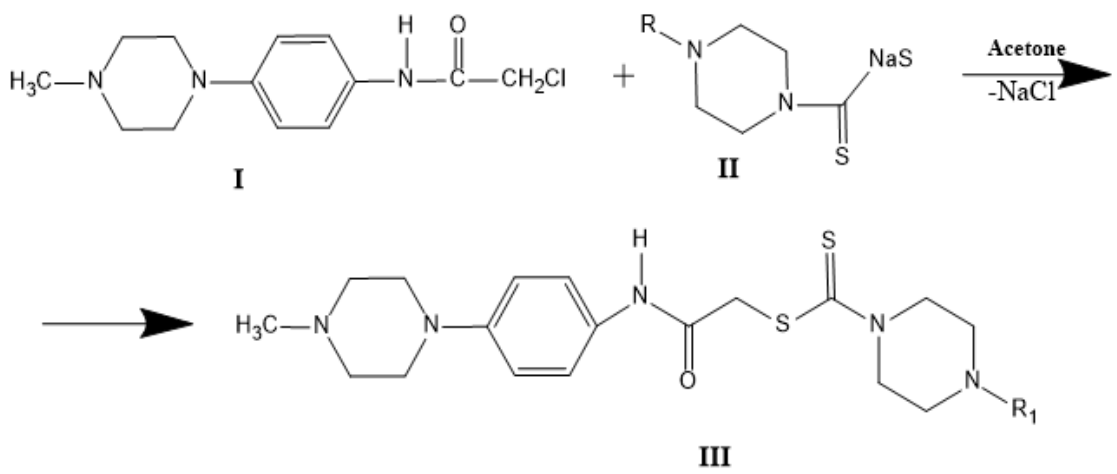

$\mathrm{R}=\mathrm{R}_{1}=\mathrm{CH}_{3}$ - substance A4 ; $\mathrm{R}=\mathrm{R}_{1}=\mathrm{C}_{2} \mathrm{H}_{5}$ - substance A5

Scheme 2. Final step of the synthesis of dithiocarbamate derivatives (A4 and A5)

\section{Substance J1:}

2-((4-morpholinophenyl)amino)-2-oxoethyl-4(4-methylbenzyl)piperazine-1-carbodithioate: Chemical formula: $\mathrm{C}_{25} \mathrm{H}_{32} \mathrm{~N}_{4} \mathrm{O}_{2} \mathrm{~S}_{2}$. Yield: $79 \%$. Melting point $145.5^{\circ} \mathrm{C}$. IR $\left(\mathrm{KBr}_{\max } \mathrm{cm}^{-1}\right): 3292$ $(\mathrm{N}-\mathrm{H}), \quad 2852-2767 \quad$ (Aliphatic $\mathrm{C}-\mathrm{H}), \quad 1666.5$ $(\mathrm{C}=\mathrm{O}), \quad 1514-1473 \quad(\mathrm{C}=\mathrm{N}$ və $\mathrm{C}=\mathrm{C}), 813 \quad(1,4-$ disubstituted benzene cycle). ${ }^{1} \mathrm{H}$ NMR $(500 \mathrm{MHz}$, DMSO- $\left.d_{6}\right): \quad \delta \quad 2,3-4.63 \quad(24 \mathrm{H}, \quad \mathrm{m}, \quad$ Aliphatic protons), 6.89 ( $2 \mathrm{H}, \mathrm{d}$, Aromatic protons, J=9.04 $\mathrm{Hz}), 7.14(2 \mathrm{H}$, d, Aromatic protons, J=7.88 Hz), 7.20 (2H, d, Aromatic protons, J=7.92 Hz), 7.43 $(2 \mathrm{H}, \mathrm{d}$, Aromatic protons, $\mathrm{J}=9.03 \mathrm{~Hz}), 10,04(\mathrm{H}$, s, NH). MS(ES): M+1:422. Element analysis: Calculated (\%); C, 56.97; H, 7.41; N, 16.61. Found (\%); C, 56.78; H, 7.32; N, 16.54.

\section{Substance J2:}

2-((4-morpholinophenyl)amino)-2-oxoethyl-4benzylpiperazine-1-carbodithioate: Chemical formula: $\mathrm{C}_{24} \mathrm{H}_{30} \mathrm{~N}_{4} \mathrm{O}_{2} \mathrm{~S}_{2}$. Yield: $82 \%$. Melting point $148^{\circ} \mathrm{C}$. IR $\left(\mathrm{KBr} \mathrm{v}_{\max } \mathrm{cm}^{-1}\right): 3292(\mathrm{~N}-\mathrm{H})$, 2852-2767 (Aliphatic C-H), $1666.5(\mathrm{C}=\mathrm{O}), 1514$ $1474(\mathrm{C}=\mathrm{N}$ və $\mathrm{C}=\mathrm{C}), 814 \quad$ (1,4-disubstituted benzene cycle). ${ }^{1} \mathrm{H}$ NMR (500 MHz, DMSO- $\left.d_{6}\right)$ : $\delta$ 2,26-4.37 (24H, m, Aliphatic protons), 6.89 $(2 \mathrm{H}, \mathrm{d}$, Aromatic protons, J=9.02 Hz) $7.15(2 \mathrm{H}$, $\mathrm{d}$, Aromatic protons, J=7.88 Hz), $7.21(2 \mathrm{H}, \mathrm{d}$, Aromatic protons, J=7.90 Hz), $7.43(2 \mathrm{H}, \mathrm{d}$, Aromatic protons, J=9.07 Hz), 10,04 (H, s, NH). MS(ES): M+1:471. Element analysis: Calculated (\%); C, 61.25; H, 6.42; N, 11.90. Found (\%); C, $61.06 ; \mathrm{H}, 6.35 ; \mathrm{N}, 11.79$.

\section{Substance A4:}

2-((4-(4-methylpiperazin-1-yl)phenyl)amino)-2oxoethyl-4-methylpiperazine-1-carbodithioate: Chemical formula: $\mathrm{C}_{19} \mathrm{H}_{29} \mathrm{~N}_{5} \mathrm{OS}_{2}$. Yield: $81 \%$. Melting point $196{ }^{\circ} \mathrm{C}$. IR $\left(\mathrm{KBr} \mathrm{V}_{\max } \mathrm{cm}^{-1}\right): 3254$ (N-H), 2918-2848 (Aliphatic C-H), 1657 (C=O), 
1514-1415 ( $\mathrm{C}=\mathrm{N}$ və $\mathrm{C}=\mathrm{C}), 820$ (1,4-disubstituted benzene cycle). ${ }^{1} \mathrm{H}$ NMR (500 MHz, DMSO- $d_{6}$ ): $\delta$ 2,23-4.21 (24H, m, Aliphatic protons), 6.88 $(2 \mathrm{H}, \mathrm{d}$, Aromatic protons, $\mathrm{J}=8.99 \mathrm{~Hz}), 7.41(2 \mathrm{H}$, d, Aromatic protons, J=8.99 Hz), 10,03 $(\mathrm{H}, \mathrm{s}$, NH). MS(ES): M+1:408. Element analysis: calculated (\%); C, 55.99; H, 7.17; N, 17.18. Found (\%); C, 54.83; H, 7.02; N, 17.04.

Substance A5:

2-((4-(4-methylpiperazin-1-yl)phenyl)amino)-2oxoethyl-4-ethylpiperazine-1-carbodithioate:

Chemical formula: $\mathrm{C}_{20} \mathrm{H}_{31} \mathrm{~N}_{5} \mathrm{OS}_{2}$. Yield: $84 \%$. Melting point $184^{\circ} \mathrm{C}$. IR $\left(\mathrm{KBr} \mathrm{V}_{\max } \mathrm{cm}^{-1}\right): 3264$ (N-H), 2974-2933 (Aliphatic C-H), $1658(\mathrm{C}=\mathrm{O})$, $1508-1429(\mathrm{C}=\mathrm{N}$ və $\mathrm{C}=\mathrm{C}), 825$ (1,4-disubstituted benzene cycle). ${ }^{1} \mathrm{H}$ NMR (500 MHz, DMSO- $d_{6}$ ): $\delta$ 2,29-4.21 (24H, m, Aliphatic protons), 6.88 $(2 \mathrm{H}, \mathrm{d}$, Aromatic protons, J=9.05 Hz), $7.42(2 \mathrm{H}$, $\mathrm{d}$, Aromatic protons, J=9.02 Hz), 10,03 $(\mathrm{H}, \mathrm{s}$, $\mathrm{NH})$. MS(ES): M+1:422. Element analysis: Calculated (\%); C, 56.97; H, 7.41; N, 16.61. Found (\%); C, 56.78; H, 7.32; N, 16.54 .

\section{Biological activity screening Anticandidal assay}

It should be noted that synthesized products were tested for their in vitro growth inhibitory activity against human pathogenic, such as Candida albicans, Candida crusei, Candida parapsilosis and Candida glabrata. Fluconazole was used as a positive control factor. Anticandidal activity test was performed according to CLSI reference M27-A3 broth microdilution method [8]. Compounds were dissolved in DMSO. Further dilutions of the compounds and standard drug in test medium were prepared in the required quantities of 1600 , $800,400,200,100,50,25,12.5,6.25,3.125$ and $1.5625 \mu \mathrm{g} / \mathrm{mL}$ concentrations with MuellerHinton broth and Sabouroud dextrose broth. In order to ensure that the solvent percent had no effect on yeast growth, a control test was also performed in calculated broth supplemented with only DMSO in the same dilutions used in our experiments and found inactive in culture medium. $\mathrm{MIC}_{50}$ and $\mathrm{MIC}$ parameters of synthesized compounds were calculated (Table 1.). Also, inhibition of new derivatives against Candida species was illustrated (Diagrams 1 and 2). Compound $\mathbf{J} 2$ showed the same effect against Candida albicans, Candida crusei, Candida parapsilosis as fluconazole. Compound $\mathbf{A 4}$ revealed the same effect against Candida parapsilosis and Candida glabrata as standard drug.

Table 1. $\mathrm{MIC}_{50}$ and MIC parameters of synthesized compounds.

\begin{tabular}{|l|l|l|c|}
\hline \multirow{4}{*}{ Compound } & \multicolumn{1}{|c|}{ Fungi species } & \multicolumn{1}{c|}{$\begin{array}{c}\text { Minimum } \\
\text { inhibitory } \\
\text { concentration } \\
50\left(\mathrm{MIC}_{50}\right)\end{array}$} & $\begin{array}{c}\text { Results of } \\
\text { minimum } \\
\text { inhibitory } \\
\text { concentration }\end{array}$ \\
\hline \multirow{3}{*}{$\begin{array}{l}\text { 2-((4-morpholinophenyl)amino)-2-oxoethyl- } \\
\text { 4-benzylpiperazine-1-carbodithioate (J2) }\end{array}$} & Candida albicans & $49.6 \mu \mathrm{q} / \mathrm{ml}$ & $100 \mu \mathrm{q} / \mathrm{ml}$ \\
\cline { 2 - 4 } & Candida crusei & $36.8 \mu \mathrm{q} / \mathrm{ml}$ & $100 \mu \mathrm{q} / \mathrm{ml}$ \\
\cline { 2 - 4 } & Candida parapsilosis & $37.57 \mu \mathrm{q} / \mathrm{ml}$ & $100 \mu \mathrm{q} / \mathrm{ml}$ \\
\cline { 2 - 4 } & Candida glabrata & $78.09 \mu \mathrm{q} / \mathrm{ml}$ & $200 \mu \mathrm{q} / \mathrm{ml}$ \\
\hline $\begin{array}{l}\text { 2-((4-morpholinophenyl)amino)-2-oxoethyl- } \\
\text { 4-(4-methylbenzyl)piperazine-1- } \\
\text { carbodithioate (J1) }\end{array}$ & Candida albicans & $78.78 \mu \mathrm{q} / \mathrm{ml}$ & $200 \mu \mathrm{q} / \mathrm{ml}$ \\
\cline { 2 - 4 } & Candida crusei & $77.15 \mu \mathrm{q} / \mathrm{ml}$ & $200 \mu \mathrm{q} / \mathrm{ml}$ \\
\cline { 2 - 4 } & Candida parapsilosis & $74.08 \mu \mathrm{q} / \mathrm{ml}$ & $200 \mu \mathrm{q} / \mathrm{ml}$ \\
\cline { 2 - 4 } & Candida glabrata & $77.16 \mu \mathrm{q} / \mathrm{ml}$ & $200 \mu \mathrm{q} / \mathrm{ml}$ \\
\hline \multirow{2}{*}{$\begin{array}{l}\text { 2-((4-(4-methylpiperazin-1- } \\
\text { yl)phenyl)amino)-2-oxoethyl } \\
\text { methylpiperazine-1-carbodithioate (A4) }\end{array}$} & Candida albicans & $76.71 \mu \mathrm{q} / \mathrm{ml}$ & $200 \mu \mathrm{q} / \mathrm{ml}$ \\
\cline { 2 - 4 } & Candida crusei & $75.58 \mu \mathrm{q} / \mathrm{ml}$ & $200 \mu \mathrm{q} / \mathrm{ml}$ \\
\hline
\end{tabular}




\begin{tabular}{|c|c|c|c|c|}
\hline & & Candida parapsilosis & $34.85 \mu \mathrm{q} / \mathrm{ml}$ & $100 \mu \mathrm{q} / \mathrm{ml}$ \\
\hline & & Candida glabrata & $37.48 \mu \mathrm{q} / \mathrm{ml}$ & $100 \mu \mathrm{q} / \mathrm{ml}$ \\
\hline 2-((4-(4-methylpiperazin-1- & & Candida albicans & $71.24 \mu \mathrm{q} / \mathrm{ml}$ & $200 \mu \mathrm{q} / \mathrm{ml}$ \\
\hline ethylpiperazine-1-carbodithioate (A5) & 4 & Candida crusei & $76.93 \mu \mathrm{q} / \mathrm{ml}$ & $200 \mu \mathrm{q} / \mathrm{ml}$ \\
\hline & & Candida parapsilosis & $35.44 \mu \mathrm{q} / \mathrm{ml}$ & $100 \mu \mathrm{q} / \mathrm{ml}$ \\
\hline & & Candida glabrata & $78.57 \mu \mathrm{q} / \mathrm{ml}$ & $200 \mu \mathrm{q} / \mathrm{ml}$ \\
\hline
\end{tabular}

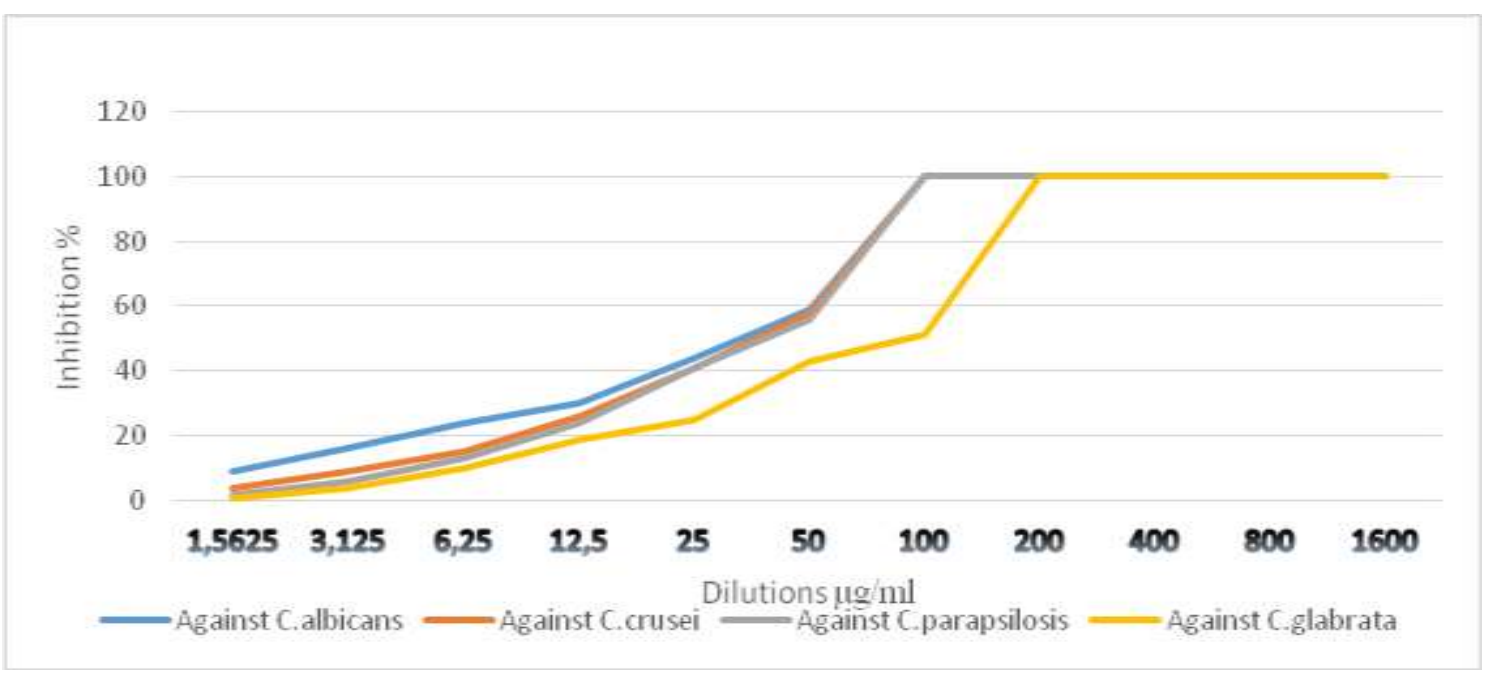

Diagram 1. Inhibition of Candida species by the compound 2-((4-morpholinophenyl) amino)-2oxoethyl 4-benzylpiperazine-1-carbodithioate (J2)

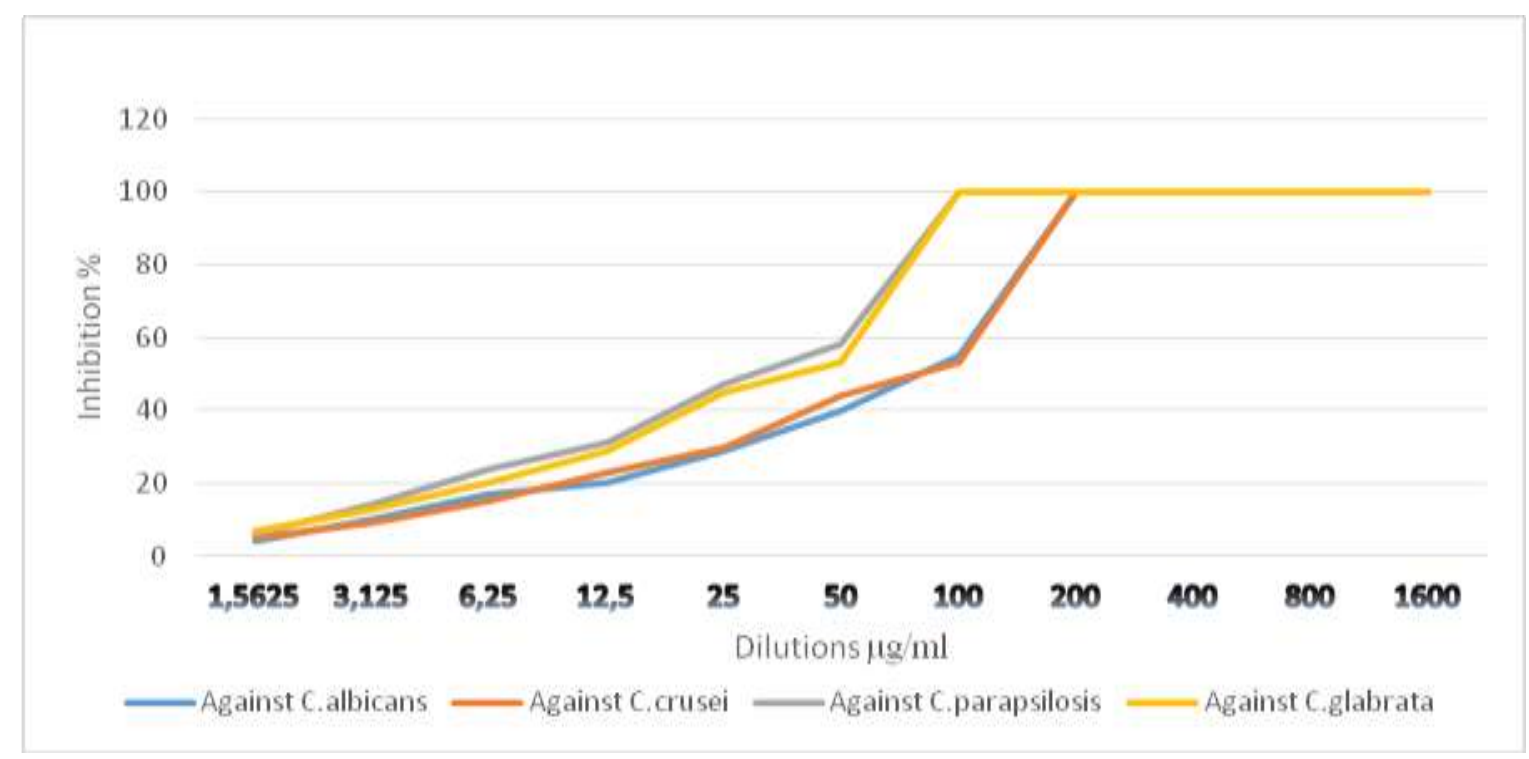

Diagram 2. Inhibition of Candida species by the compound 2-((4-(4-methylpiperazin-1-yl) phenyl) amino)-2-oxoethyl 4-methylpiperazine-1-carbodithioate (A4) 


\title{
Conclusion
}

New derivatives of piperazine-1dithiocarbamate with morpholineaniline ( $\mathbf{J} 1$ and J2) and with N-methyl piperazineaniline (A4 and A5) were synthesized and their structures identified. The biological activity of synthesized derivatives of dithiocarbamates were examined, $\mathrm{MIC}_{50}$ and MIC parameters of synthesized compounds were calculated and reveal antifungal activity of the compounds $\mathbf{J} 2$ and $\mathbf{A 4}$.

\section{References}

1. Lalit K., Nand L., Vikash K. et all. Azolecarbodithioate hybrids as vaginal antiCandida contraceptive agents: Design, synthesis and docking studies. European Journal of Medicinal Chemistry, 2013, vol. 70, pp. 68-77.

2. Monti M.M., Maresca A., Viparelli F. Dithiocarbamates are strong inhibitors of the beta-class fungal carbonic anhydrases from Cryptococcus neoformans, Candida albicans and Candida glabrata. Bioorganic \& Medicinal Chemistry Letters, 2012, vol. 22, issue 2, pp. 859-862.

3. Chauhan K., Sharma M., Singh P., et al. Discovery of a new class of dithiocarbamates and rhodanine scaffolds as potent antifungal agents: synthesis, biology and molecular docking. Med.Chem.Commun., 2012, vol. 3, pp. 1104-1110.

4. Panneerselvam P., Nair R.R., Vijayalakshmi G., Subramanian E.H., Sridhar S.K., Synthesis of Schiff bases of 4-(4-aminophenyl)morpholine as potential antimicrobial agents. European Journal of Medicinal Chemistry. 2005, vol.40, pp. 225-229.
5. Chaudhary P., Kumar R., Verma A.K. Synthesis and antimicrobial activity of $\mathrm{N}$-alkyl and $\mathrm{N}$-aryl piperazine derivatives. Bioorganic \& Medicinal Chemistry, 2006, vol. 14, pp. 1819-1820.

6. Khanum S.A., Begum B.A., Girish V., Khanum N.F. Evaluation of Benzophenone-Nethyl Morpholine Ethers as Antibacterial and Antifungal Activities. International Journal of Biomedical Science, 2010, vol. 6, no.1, pp. 6065.

7. Dhahagani K., Mathan K.S., Chakkaravarthi G. Synthesis and spectral characterization of Schiff base complexes of $\mathrm{Cu}(\mathrm{II}), \mathrm{Co}(\mathrm{II}), \mathrm{Zn}$ (II) and $\mathrm{VO}(\mathrm{IV}) \quad$ containing 4-(4aminophenyl)morpholine derivatives: Antimicrobial evaluation and anticancer studies. Spectrochimica Acta Part A: Molecular and Biomolecular Spectroscopy. 2014, vol. 117, pp. 87-94.

8. Reference method for broth dilution antifungal susceptibility testing of yeasts; approved standard-third edition; CLSI document M27A3, Clinical and Laboratory Standards Institute. 2008.

\section{YENI DITIOKARBAMAT TÖRӘMӘLӘRININ SINTEZI VӘ BIOLOJI FOALLIĞI}

\author{
Ç.Y. Şükürrov \\ Azarbaycan Tibb Universiteti, aczaçılıq kimyası kafedrası \\ AZ 1022, Bakl, S.Vurğun küç., 167, e-mail: china.86@mail.ru
}

Hal-hazırda bir çox müxtəlif göbələk aleyhinə vasitalar vardır. Bunların arasında ditiokarbamatlar töramalari da var. Bu işin maqsadi yeni ditiokarbamat töramalarinin sintezi va antifunqal faallığının öyranilməsidir. Sintez olunmuş maddəlarin tədqiqi və strukturlarının müayyənləşdirmək üçün aşağıdakı spektral metodlardan istifado edilmişdir: DMSO-d6-da IR (Shimadzu 8400 FTIR), IH NMR (Bruker 
$500 \mathrm{MHz}$ UltraShield) (daxili standart - tetrametilsilan), Mass spektroskopiya (Agilent 1100 Series LC) / MSD Trap VL \& SL). Element analizi (C, H vo N) Perkin Elmer analizatoru vasitosi ilo aparılmışdır. Sintez olunmuş ditiokarbamat törəməlorinin bioloji faalliğ t tədqiq edilmiş, J2 birloşməsinin $100 \mathrm{mkq} /$ ml konsentrasiyada durulaşmada Candida albicans, Candida crusei, Candida parapsilosis ştamlarına qarşı flukonazol ilə müqayisədə eyni faallıq nümayiş etdirmişdir. A4 maddəsi, standart nümunə ilə (flükonazol) müqayisədə Candida parapsilozu vo Candida glabrata ştamlarına eyni faallı̆̆l göstarmişdir.

Açar sözlor: ditiokarbamatlar törəməlari, antifunqal faallı̆̆

\section{СИНТЕЗ И БИОЛОГИЧЕСКАЯ АКТИВНОСТЬ НОВЫХ ПРОИЗВОДНЫХ ДИТИОКАРБАМАТОВ}

\section{Ч.Я. Шукюров}

Азербайджанский медицинский университет, кафедра фармацевтической химии AZ 1022, Баку, ул. С.Вургуна, 167, e-mail: china.86@mail.ru

В настоящее время существует множество различных противогрибковых средств, среди которых есть и производные дитиокарбаматов. Целью данной работы является синтез новых производных дитиокарбаматов и изучение их противогрибковой активности. Для анализа $и$ идентификации синтезированных вещчеств $u$ определения их структурных формул использовались следующие спектральные методы: ИК (Shimadzu 8400 FTIR), 1H ЯMP (Bruker $500 \mathrm{MHz}$ UltraShield) в ДМСО-d6 (внутренний стандарт - тетраметилсилан), Массспектроскопия (Agilent 1100 Series LC / MSD Trap VL \& SL). Элементный анализ (C, H и N) проводили с использованием анализатора Perkin-Elmer. Была исследована биологическая активность синтезированных производных дитиокарбаматов и соединение J2 (2 - ((4морфолинофенил) амино) -2-оксоэтил 4-бензилпиперазин-1-карбодитиоат) при концентрации 100 мкг / мл показало такую же активность в отношении Candida albicans, Candida crusei, Candida parapsilosis, что и флуконазол. Соединение A4 (2 - ((4-(4-метилпиперазин-1-ил) фенил) амино) -2-оксоэтил 4-метилпиперазин-1-карбодитиоат) проявило активность в отношении Candida parapsilosis и Candida glabrata, что и стандартное лекарственное средство.

Ключевые слова: противогрибковые средства, производные дитиокарбаматов 